\title{
An approach for smart parking system based on cloud using IoT
}

\author{
Swapna S. Deshpande \\ ME Student \\ Department of Information Technology \\ Pimpri Chinchwad College of Engineering \\ Pune, India
}

\author{
Prof.Renuka S. Gound \\ Assistant Professor \\ Department of Information Technology \\ Pimpri Chinchwad College of Engineering \\ Pune, India
}

\begin{abstract}
- in the most of the modern cities it is difficult and expensive to create more parking spaces for vehicles since the numbers of vehicles are running on the road are increasing day by day and the count of the free spaces in the cities are the same. This problem leads to congestion for parking seekers and drivers. To develop an IoT framework that targets Parking Management which is biggest challenges in modern cities. Using embedded systems, there is a chance to develop an application which can solve these problems. The proposed smart parking solution gives an onsite deployment in which, IoT application monitors and indicate the availability of each parking space. This system helps in improvising the management of parking system by following rules of the government, for example handling different parking spaces in the city. The intuition of presenting this paper is to reduce smart city issue such as the traffic on road and reduces the pollution in the city and the parking.
\end{abstract}

Keywords-Cloud; Internet of Things; MQTT; Raspberry pi; Parking

\section{INTRODUCTION}

Currently, the IoT applications in our daily life are blooming, and there is also a growing trend in the applications of smart cities which can help in improving to reduce smart cities issues. In Smart City we faces many difficulties while developing, to solve smart city issues we have to develop such system which is combination of the new technology also of low cost and based on the different network combination of the Internet, such as a telecommunications, broadcast, wireless and sensor networks where Internet of Things (IoT) is base technology. One of the major issues in a smart city is the Parking. A parking lot should provide customers enough spaces to park their car since car plays a huge role in transportation, there is need of finding out parking area to park the vehicles. By creating a new system, it can help manage and reducing the road traffic. A new system helps customers to save time in finding a parking spot. The Internet of Things is about installing different sensors like ultrasonic sensors; active and passive RFID, IR, etc. connect to the internet through different protocols for exchanging information and to communicate, in order to achieve monitoring, management. Using IoT, Smart City can be established by integrating these features for IoT development. The Internet of Things (IoT) uses devices which are connected to each other and systems to collect the data by using embedding sensors, actuators and other physical objects. Rest this paper is organized as follows. Section II shows related work on smart parking system.
Section III contains the proposed smart parking system and Section IV contains conclusion followed by references.

\section{RELATED WORK}

There are a number of applications that give information about free parking slot to users. All the currently running application gives an interface through a mobile phone, and desktops applications. They mainly focus on finding the cheapest and nearest parking lot through Google maps and they do not provide information about spot availability (Gupte \& Younis, 2015; Rico, Sancho, Cendón, \& Camus, 2013) There are different models for parking management developed per year, mostly they consist of RFID-based, and wireless sensor based methods. These methods track the free space. The RFID tags are put on vehicles to take down the in-out time of the vehicle based on that cost for parking is decided. However, a disadvantage of such RFID method is it is a bit expensive to keeping track of RFID tags, and in cases of wireless sensors, various sensors are used are like weight, motion which is difficult to maintain (Gupte \& Younis, 2015). The cloud system used to store the data from each sensor on a daily basis, thus cloud provides unlimited storage capacity, which is low in cost and it has ondemand storage capacity (Khanna, 2016). In a traditional way we have to install wired infrastructure for vehicle detection, but now a day's using wireless sensor network through Zigbee networks we can install an infrastructure. Zigbee network wirelessly communicates with a central server that has all information about the sensor's data (Grodi \& Rios-gutierrez, 2016). In this paper, they implement a parking guidance system using RTOS and PIC32 controller. In that one microcontroller controls the three sensors at a time (Yosafat, 2014) they have used Free RTOS and RS485 communication protocol for transmitting and receiving data. Raspberry pi board contains System on a Chip (SoC) it means it is a method of grouping all the useful electronics to run on the individual chip and it also uses processor Broadcom BCM2837 for various interfaces. Raspberry pi is flexible; it can use for general purpose computing. (Vujović \& Maksimović, 2014). In this paper, they have created parking system based on the wireless communication network which efficiently manages time to find out parking space in less time [7]. In this paper, they have created zone area to control over each parking lane and all zones are connected 
through central control unit [9] this paper they have proposed parking system using magnetic sensors[10]

\section{III.PROPOSED SYSTEM}

The proposed system consists of three main phases:
A. On-Field Network
B. Cloud Platform
C. User side Platform

\section{A. On-Field Network}

As shown in figure 1, On-Field Network section there will be one ultrasonic sensor for individual parking slot is allotted, which will be ultimately collect the data from each parking slot. Following are the devices we are going to used on field network.

\section{Ultrasonic sensor:}

A basic ultrasonic sensor consists of a transmitter, a receiver, and a control circuit. The transmitter emits a high-frequency ultrasonic sound, which bounces off any nearest solid objects. The ultrasonic sound has detected the obstacle which is within the range and reflects the signal to the sensor. That reflected signal is then processed by the control circuit to calculate the time interval between the signal transmitted and received. Ultrasonic sensors are designed to sense range using ultrasound reflection, like the radar system, which calculates the time interval between the sensor and a solid object by passing ultrasonic waves. Ultrasonic sound is mainly used since it is inaudible to the human ear and it gives almost accurate results within short distances.

\section{ESP8266 Chip:}

An ESP8266 chip is the Wi-Fi chip, which is low in cost. It is a small module which allows microcontrollers to connect to the WiFi network.

\section{Raspberry Pi:}

Raspberry pi 3 model B is a single board computer which is of credit card sized. the system on chip It has several versions model A, A+, B, B+, zero. Raspberry pi board contains System on a Chip (SoC) it means it is a method of grouping all the useful electronics to run on the individual chip and it also uses processor Broadcom BCM2837 for various interfaces, a program memory (RAM) is of 1GB. Raspberry pi used as a computer requires a keyboard for entering commands, display as well as the power supply of $2.5 \mathrm{~A}$. The Raspberry pi 3 model $\mathrm{b}$ is most efficient board.

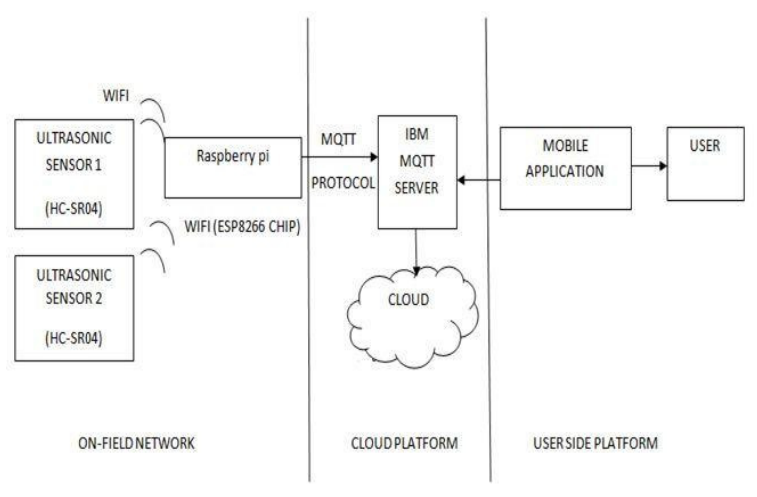

Figure 1: Proposed Smart Parking System

\section{B. Cloud Platform}

\section{MQTT MQ Telemetry Transport:}

MQTT described as Machine to Machine (M2M)/ IoT connectivity protocol. This protocol is light weighted; the protocol can be supported by smallest measuring and monitoring device. MQTT is publish-subscribe messaging transport protocol that helps to connect physical devices to the servers. There are many challenges of connecting sensors, actuators, mobile phones, tablets and desktops with established software technologies, MQTT designed to overcome these problems. The MQTT messages are delivered asynchronously through publish-subscribe architecture. It works by exchanging a series of MQTT control packets in a defined way. The control packet sent over the network has a specific purpose and every bit in the packet is carefully crafted to reduce the data transmission an MQTT topology has an MQTT server and an MQTT client. MQTT client and server are communicating through different control packets. MQTT control packet headers are kept as small as possible. An individual MQTT control packet divides into three parts, a fixed header, a variable header, and the payload. Fixed header for each MQTT control packet is 2 bytes. Some of the control packets have variable headers and payload. A variable header contains packet identifier when it is used by the control packet. The packets can be attached payload up to $256 \mathrm{MB}$.

\section{User Side Platform}

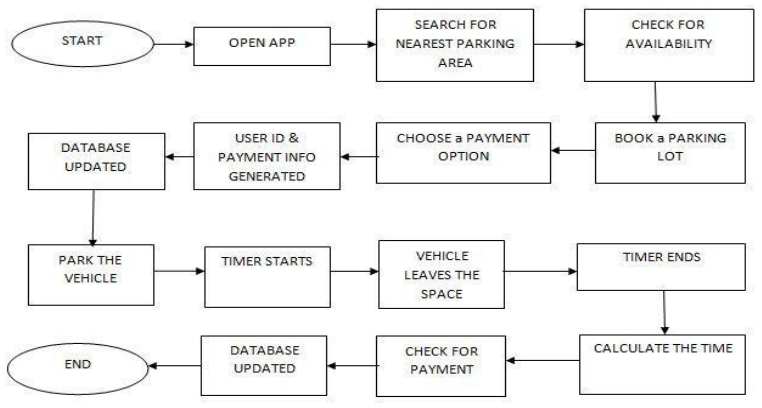

Figure 2: Data flow diagram of User Side Platform

As shown in figure 2, in User Side Platform section, the user will open the app, he will search for the nearest parking area, it checks for availability of free parking lot. 
If the parking lot is empty, the user will book the lot. After that he chooses for payment option then user id and payment information will be generated. The information about user and payment is updated in the database. The user will park the vehicle, and the timer will start when the user leaves the space timer will be stopped. The time is calculated, if the time exceeds than allotted time then the user have to pay once again and the database is updated that slot is free for parking.

\section{CONCLUSION}

In the smart cities, mainly people face problems like parking issue, traffic congestion, this paper analyses different smart parking system available in literature and also proposes a solution based on the cloud. Using internet of things in a smart parking system it helps in reduction in consumption of fuel, it reduces traffic congestion in cities and cloud used for storing the information which is collected from the sensors.

\section{REFERENCES}

[1] Gupte, S., \& Younis, M. (2015). Participatory-sensing-enabled Efficient Parking Management in Modern Cities, 241-244.

[2] Rico, J., Sancho, J., Cendón, B., \& Camus, M. (2013). Parking easier by using context information of a Smart City Enabling fast search and management of parking resources, 1380-1385. https://doi.org/ 10.1109/WAINA.2013.150

[3] Khanna, A. (2016). IoT based Smart Parking System, 266-270.

[4] Grodi, R., \& Rios-gutierrez, F. (2016). Smart Parking: Parking Occupancy Monitoring and Visualization System for Smart Cities, 1-5.

[5] Yosafat, J. (2014). Parking Guidance System Based on Real Time Operating System, (August), 28-31.

[6] Vujović, V., \& Maksimović, M. (2014). Raspberry Pi as a Wireless Sensor node: Performances and constraints. 2014 37th International Convention on Information and Communication Technology, Electronics and Microelectronics, MIPRO 2014 - Proceedings, (May), 1013-1018. https://doi.org/10.1109/MIPRO.2014.6859717

[7] Srikanth, S. V., Pramod, P. J., Dileep, K. P., Tapas, S., Patil, M. U., \& Babu N, S. C. (2009). Design and implementation of a prototype smart parking (SPARK) system using wireless sensor networks. Proceedings International Conference on Advanced Information Networking and Applications, AINA, 401-406. https://doi.org/10.1109/WAINA.2009. 53

[8] Kianpisheh, A., Mustaffa, N., Limtrairut, P., \& Keikhosrokiani, P. (2012). Smart Parking System (SPS) architecture using ultrasonic detector. International Journal of Software Engineering and Its Applications, 6(3), 51-58.

[9] Boda, V. K., Nasipuri, A., \& Howitt, I. (2007). Design considerations for a wireless sensor network for locating parking spaces. Conference Proceedings - IEEE SOUTHEASTCON, 698-703. https://doi.org/ 10.1109/SECON.2007.342990 